them in a dictionary designed for everyday use. Still, the terms on omission of which we have commented are important and in common use, and it is deeply to be desired that in future editions this side of the dictionary will be thoroughly overhauled.

The value of this dictionary is not to be gauged in terms of the three guineas of its price. Words are the counters of wise men and the money of fools; and the world would be saved much trouble and strife if its inhabitants were only more skilled. in the use of these counters. To the increase of that skill our new dictionary is destined to contribute even more largely than the parent lexicon, and the perfecting of this instrument, already most valuable, is a matter of immediate concern to all. Such alterations and additions as may be necessary to be made deal merely with matters of detail ; and even as a Lover of the Arts said of that Compleat Dictionary printed at Cambridge by John Field in 1667, so we may heartily recommend this product of twentieth century erudition to "those vertuous and well-addicted Persons who . . have here a volume fit for their purpose, as carefully designed for their assistance: and to such, and onely such, we recommend it, and that with this Benediction, Live long, industrious Reader, advance in Knowledge, and be happy."

\title{
Deep-Sea Angler Fishes
}

$\mathrm{T}$ HERE still remains a large field for the discovery of new forms of animal life in the deeper layers of the ocean water masses. There, pelagic life is not concentrated at one level, but has a vertical range of many hundreds of metres in which to roam as well as thousands of square miles horizontally. The larger animals thus tend to become comparatively sparsely distributed. To sample this deep-living population, great quantities of water must be filtered, and it is only to be expected that the recent introduction of large pelagic nets would bring a considerable extension to our knowledge. Perhaps nowhere is this better emphasised than in the study of the deep-sea angler fishes of the sub-order Ceratioidea; as a result of the examination of the collection of these fishes made on the world voyage of the Dana in 1928-30 under the late Prof. Johannes Schmidt, our knowledge has been amplified and brought up to date by Dr. C. Tate Regan and Miss Ethelwynn Trewavas*.

Although a systematic revision of the group was made by Dr. Tate Regan in 1926, the addition of new species and collection of new specimens has been so great that the authors have found it necessary to make a further revision. In the former report the number of species was 60 while now 158 species are recognised. Previous collections had consisted, to a considerable extent, of specimens less than one inch in length which had been assigned to those known species which they most nearly resembled; it is now shown that, among the Oneirodidæ, the adult characters may be assumed at a very small size.

The Ceratioids have recently become known in the zoological world on account of the discovery, on the females of certain species, of dwarfed parasitic males the bodies of which had become fused to that of the female at an early stage. This was known in the four families, Ceratias, Photocorynus, Edriolychnus, and Caulophryne. The same habit has now been shown to occur also in the families Borophryne and

* The Carlsberg Foundation's Oceanographical Expedition round the World, 1928-30. Report No. 2: Deep-Sea Angler-Fishes (Ceratioidea). By Dr. C. Tate Regan and Ethelwynn Trewavas. (Published by the Carlsberg Foundation.) Pp. 113 +10 plates. (Copenhagen : C. A. Reitzels Forlag ; London: Oxford University Press, 1932.) 158. net.
Linophryne; the authors also say that the continuity of the blood systems of the male and female has been proved in Edriolychnus.

As only females of the solitary adult type had been found in previous collections, it was thought that perhaps the habit of parasitisation by the males was of general occurrence throughout the sub-order. In 1930, however, Parr recorded, the discovery of a free-living male (Rhyncoceratias longipinnis) with a well-developed testis. In the report before us it is now shown also that the males are not parasitic in the Melanocetidæ, Himantolophidæ, and Oneirodidæ. They are small free-swimming fish which show considerable structural differences from the females. They have no illicium, have large olfactory organs, and except in two naked species, the skin is spinulose.

Typically the female ceratioids have large mouths, strong teeth and very distensible stomachs; they are solitary and inactive and by means of their luminous lures (the illicium) they attract prey often larger than themselves. The males have small mouths and stomachs and probably swim actively in search of their prey and of their mates. It is thought that the females most nearly retain the structure and habits of the original members of the group, since they possess the illicium so characteristic of angler fishes. They appear normally to inhabit the layers of the ocean at 500-2000 m. depth and are most abundant in warmer parts of the ocean. The comparison of ceratioids from the Panama region with those of other regions of the Pacific needs further study.

A section of the report is devoted to the osteology and classification of the sub-order. The most important structures for diagnosing the families are, the structure of the fins, form of the hyomandibular, number of branchiostegals, number of pectoral radials, and presence or absence of pelvic bones. The last and greater part of the report is taken up with the systematic revision. The volume contains also ten plates of illustrations of 22 species by Lieut.-Col. W. P. C. Tenison, which emphasise how effective stippling can be in the representation of solid form, where the expense of reproduction of wash drawing seems unnecessary. F. S. R. 